\title{
Retrospective analysis of epsilon-amino-caproic acid (EACA) Therapy in patients with hypoproliferative thrombocytopenia
}

\begin{abstract}
Bleeding still contributes to significant morbidity and mortality in patients with hypoproliferative thrombocytopenia even with optimal platelet transfusion support. We hypothesized that Epsilon-amino-caproic acid (EACA) therapy might stabilize a hemostatic plug thereby reducing bleeding in these patients. Over six years at our hospital, data from 133 patients with hematologic malignancies were retrospectively analyzed for three days prior to and for up to seven days after starting EACA therapy. Ninety-nine of these patients $(74 \%)$ had active bleeding and with EACA therapy, bleeding decreased in $66 \%$, was unchanged in $26 \%$ and increased in $8 \%$. Even in the 45 patients with severe bleeding (WHO Grades 3 or 4), $62 \%$ showed bleeding improvement and in 6 with intra-cerebral bleeding, 3 improved and 3 stabilized. In the remaining 34 patients treated prophylactically (26\%), usually because of refractoriness to platelet transfusions, $91 \%$ had no bleeding and $9 \%$ had only WHO Grade 1 bleeding. Among all treated patients, platelet and red cell transfusions decreased significantly by 0.25 and 0.24 units transfused/patient/day $(p<0.003$ and $\mathrm{p}<0.001$ respectively) compared to blood product requirements before EACA therapy. These data suggest that EACA therapy may be a useful adjuvant to treat or prevent bleeding in thrombocytopenic patients.
\end{abstract}

Keywords: thrombocytopenia, epsilon-amino-caproic acid, platelet transfusions, red cell transfusions, bleeding
Volume 4 Issue I - 2017

\author{
Gernsheimer T,' Bolgiano D, ${ }^{2}$ Slichter SJ ${ }^{3}$ \\ 'Department of Hematology, University of Washington, USA \\ 2BloodWorks Northwest, USA \\ ${ }^{3}$ Department of Hematology \& BloodWorks Northwest, \\ University of Washington, USA
}

Correspondence: Terry Gernsheimer, Division of Hematology, University of Washington, 1959 NE Pacific Street, Box 356330, Seattle,WA, 98195, USA, Tel 206-598-9066, Fax 206-598-4021, Email bldbuddy@u.washington.edu

Received: January 19, 2017 | Published: January 26, 2017
Abbreviations: EACA, epsilon-amino-caproic acid; HSCT, hematopoietic stem cell transplantation; CT, computerized tomography; ICH, intra-cranial bleeding; $\mathrm{SDH}$, sub-dural hematoma; SAH, sub-arachnoid hemorrhage; DVT, deep venous thrombosis; cPRA, calculated panel reactivity

\section{Introduction}

Bleeding remains a significant problem in patients with hypoproliferative thrombocytopenia due to chemotherapy, radiation induced marrow aplasia and/or hematopoietic stem cell transplantation (HSCT). Prolonged periods of thrombocytopenia in these patients may require ongoing extensive platelet transfusion support. However, despite platelet transfusion therapy, three large randomized trials that closely monitored bleeding rates in patients with hematologic malignancies have demonstrated that the incidence of WHO Grade 2 or higher bleeding ${ }^{1}$ remains high at $43 \%$ to $71 \% .^{2-4}$ Bleeding rates did not appear to be improved by either increasing the platelet dose per transfusion or by increasing the prophylactic platelet transfusion threshold above 10,000 platelets $/ \mu 1,2,5,6$ suggesting that factors other than thrombocytopenia may be contributing to these patients bleeding. Clearly, other approaches to prevent and treat bleeding in thrombocytopenic patients are needed. Epsilon amino-caproic acid (EACA) is a lysine analogue that competitively inhibits plasminogen activation and acts as a non-competitive inhibitor of plasmin at high concentrations. ${ }^{7}$ Inhibitors of fibrinolysis have been used for years to prevent and treat bleeding intra- and post-operatively. ${ }^{8}$ Fibrinolytic inhibitors have also been effective in the control of bleeding in patients with platelet function ${ }^{9}$ and coagulation defects. ${ }^{10}$ To date, there have been only a few reports of EACA administration in a small number of thrombocytopenic patients. ${ }^{11-14}$ These reports have been encouraging, with improvements in prevention and control of bleeding, but the efficacy and safety of EACA in this setting remains uncertain. We report here our retrospective analysis of the use of EACA therapy in thrombocytopenic patients undergoing chemotherapy and/or HSCT to evaluate its effects on bleeding, transfusion requirements and safety.

\section{Materials and methods}

Using a pharmacy database, we identified medical records of patients who had ever received EACA therapy, from January 2006 to July 2012, at the University of Washington Medical Center and Seattle Cancer Care Alliance hematology-oncology outpatient clinics. Inclusion criteria were patients $\geq 18$ years old who received either oral or I.V. EACA therapy for at least 24 hours. Patients were excluded if EACA was started as comfort care or the medical record was incomplete for the indications, dose, or duration of EACA therapy. Data were collected on the indication for EACA therapy (to treat bleeding versus prophylaxis to prevent bleeding), bleeding symptoms and red cell and platelet transfusion requirements for three days prior to and up to seven days after starting EACA therapy. We used a modification of the WHO bleeding scale1 to assign a bleeding grade (Table 1)

All spontaneous skin bleeds were considered Grade 1 bleeding, unless a skin laceration or line insertion site required a surgical intervention (e.g., hematoma drainage or suturing), in which case it was graded as WHO Grade 2 bleeding. If a patient had more than one bleeding site, the site with the higher bleeding score was used to assign a bleeding grade for that day. Data were also collected 
on thrombotic events for three days before therapy and for three weeks after starting EACA therapy. Data were obtained from patient records using a case report form developed before study initiation. This retrospective data review was approved by the University of Washington's Institutional Review Board. Platelet refractoriness was defined as failure to show an increase in the platelet count of at least $10,000 / \mu \mathrm{l}$ within 4 hours after receiving an apheresis platelet transfusion or a 4-6 unit pool of platelet concentrates prepared from whole blood donations. Splenomegaly was based on ultrasonography imaging, computerized tomography (CT) scan, or magnetic resonance imaging (MRI) of the abdomen; an enlarged spleen was assessed as more than $10 \mathrm{~cm}$ in its largest dimension. Class I HLA IgG antibodies were detected in the patients' sera using LABScreen single antigen assay (One Lambda, Inc., Canoga Park, CA). This assay uses micro beads coated with purified Class I HLA antigens to detect antibodies in the patient's serum using Luminex xMAP multiplex technology. Using a panel of 1,000 deceased organ donors, the percent of donors reactive with the patient's serum were used to determine the percent calculated panel reactivity (cPRA).

Table I Modified WHO bleeding scale

\begin{tabular}{|c|c|c|c|}
\hline Grade I & Grade 2 & Grade 3 & Grade 4 \\
\hline $\begin{array}{l}\text { Minor Bleeding (e.g., } \\
\text { all spontaneous } \\
\text { skin bleeds, guaiac } \\
\text { positive stool, etc.). }\end{array}$ & $\begin{array}{l}\text { Bleeding requiring: } \\
\text { I. An intervention to control bleeding (e.g., nasal packing, bladder irriga- } \\
\text { tion, invasive site bleeding requiring sutures, etc. } \\
\text { 2. Platelet transfusion to treat active bleeding; or } \\
\text { 3. Medications other than EACA given to treat bleeding e.g. progeste- } \\
\text { rone analogues for active vaginal bleeding, pantoprazole for upper } \\
\text { gastro-intestinal bleeding, etc. }\end{array}$ & $\begin{array}{l}\text { Bleeding requiring: } \\
\text { I. Significant intervention to } \\
\text { treat bleeding (e.g., endos- } \\
\text { copy or surgery); or } \\
\text { 2. Red cell transfusion related } \\
\text { to treatment of bleeding. }\end{array}$ & $\begin{array}{l}\text { Bleeding } \\
\text { that is } \\
\text { fatal or life } \\
\text { threatening. }\end{array}$ \\
\hline
\end{tabular}

\section{Results and discussion}

\section{Statistics}

Descriptive statistics are presented. The red cell transfusions (units/ patient/day) given before and after EACA therapy were reported as medians and compared using the Wilcoxon signed-rank statistic, since the data were not normally distributed. Platelet transfusions (units/patient/day) before and after EACA therapy were reported as means and compared using the student t-test because these data were normally distributed.

\section{Patient population}

We identified 221patients who had been treated with EACA therapy on 260 separate occasions and only data from the first treatment given to each patient were analyzed. Thirty-seven patients who received EACA for less than 24hours, 21patients who received EACA as comfort care, 26patients with incomplete records and 4 patients who received only non-systemic EACA (oral swish and spit formulation) were excluded from the analysis. The remaining 133 patients formed the basis for this report. The majority of the patients were undergoing therapy for a hematologic malignancy (115) or aplastic anemia (6). The other 12patients had autoimmune thrombocytopenia (8), chemotherapy induced marrow aplasia for treatment of a solid tumor (2), drug induced thrombocytopenia (1) and transfusion associated graft versus host disease (1). Of the 133 patients, 44 were post HSCT (36\%) (37 allogeneic and 7 autologous). Patient demographics are summarized in Table 2. Median age was 53 years (range 42-63years) and 47\% were male. The median platelet count at the start of EACA therapy was $12,000 / \mu 1$ (range 5000/ $\mu 1-$ $28,000 / \mu \mathrm{l}$ ) and median hematocrit was $26 \%$ (range 24\%-28\%). Transfusions were given according to standard threshold guidelines. The trigger for a prophylactic platelet transfusion was a platelet count of $10,000 / \mu \mathrm{l}$ and for a red blood cell transfusion was a hematocrit of $27 \%$. Both products could be given at higher levels for patients with active bleeding. Among the evaluable patients, 110 patients $(83 \%)$ were treated only as in-patients, 13 patients started therapy as in-patients and continued EACA therapy as outpatients (10\%) and 10 patients $(8 \%)$ were treated exclusively as outpatients.
Table 2 Demographic Data

\begin{tabular}{lll}
\hline & $\begin{array}{l}\text { Number of } \\
\text { patients }\end{array}$ & $\%$ of patients \\
\hline Males & 62 & 47 \\
Females & 71 & 53 \\
Platelet Refractory* & 74 & 56 \\
CPRA Done** & 89 & 67 \\
$\begin{array}{l}\text { CPRA**>20\% } \\
\text { Platelet Refractory, CPRA Done, And }\end{array}$ & 57 & $64 * * *$ \\
$\geq 20 \%$ Positive (Presumed Alloimmune & 52 & $39 * * *$ \\
$\begin{array}{l}\text { Refractory) } \\
\text { Splenomegaly }\end{array}$ & 40 & 30 \\
$\begin{array}{l}\text { Platelet refractory } \\
\text { Post Hematopoietic Stem Cell }\end{array}$ & 20 & $50 * * * *$ \\
Transplant & 44 & 36 \\
End Stage Renal Disease & 8 & 6 \\
\hline
\end{tabular}

* Failure to show increase in platelet count by $10,000 / \mu \mathrm{l}$ within 4 hours of a platelet transfusion.

** Calculated Panel Reactive Antibody (HLA class I antigen, microbead assay).

*** Percentage of patients tested.

**** Percentage of patients with splenomegaly who were also platelet refractory.

\section{Evaluation of actively bleeding patients}

Response to EACA therapy in actively bleeding patients: Of the 133 patients, 99(74\%) were treated with EACA for active bleeding. Their average age was 51 years, $46 \%$ were males and 33 patients $(33 \%)$ were post HSCT. The median platelet count at the start of EACA therapy was $17,000 / \mu 1$ (range $5,000 / \mu 1-32,000 / \mu l$ ) and median Hct was 26\% (range 23-29). The highest grade of bleeding before starting EACA therapy was Grade 2 in 33 patients $(33 \%)$, Grade 3 in 28 patients $(28 \%)$ and Grade 4 in 17 patients (17\%). Twenty- 
one patients had only Grade 1 bleeding. Daily EACA doses in these patients ranged from 3 to 24 grams (median 8 grams) in 3 , 4 , or 6 divided doses with one patient on a continuous infusion of 12 grams per day. Of the 99 patients treated with EACA for active bleeding, bleeding improved in $65(66 \%)$ of the patients, was unchanged in $26(26 \%)$ and worsened in $8(8 \%)$ of patients. Of the 78 patients with clinically-significant bleeding (Grade 2 or higher), $68 \%$ had improvement in their bleeding, $10 \%$ worsened and $22 \%$ remained unchanged. Sixty-two percent of the 45 patients with severe bleeding (WHO Grade 3 or higher) had improvement in their bleeding; i.e., 21 of 28 patients $(75 \%)$ with Grade 3 bleeding and 7 of the 17 patients (41\%) with Grade 4 bleeding. The highest grades of bleeding on the three days prior to EACA therapy (days -3 to -1 ) and the seven days after starting EACA therapy (days 1 to 7 ) are shown in Figure 1. There was a progressive increase in bleeding severity prior to EACA therapy (days-2 to 0 ) and a progressive decrease after treatment (days 1 to 7 ). Neither age, gender, diagnosis, platelet count or hematocrit at the start of therapy, nor EACA dose predicted response to EACA therapy (data not shown).

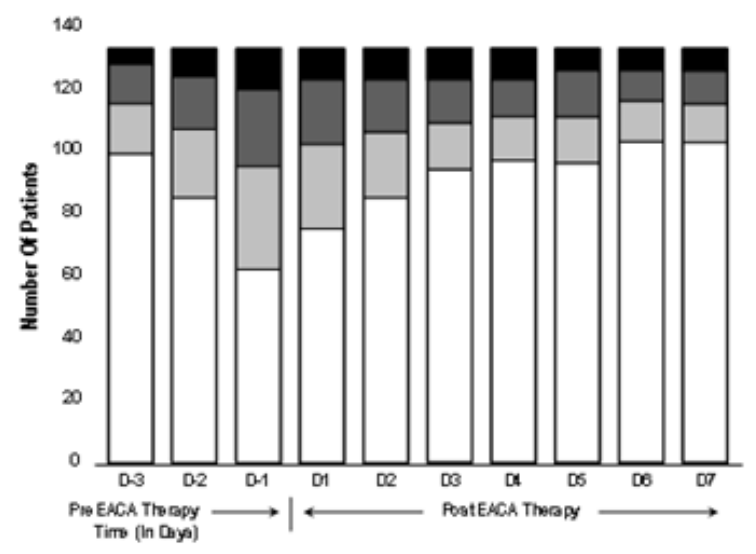

Figure I Highest grade of WHO bleeding per day.

Bleeding sites: The sites of bleeding are given in Table 3. There were 6 patients treated with EACA for intra-cranial bleeding (ICH). In three of these cases, the bleeding stabilized with EACA therapy and the neurologic symptoms remitted [sub-dural hematoma (SDH) with seizures, SDH with facial weakness, sub-Arachnoid hemorrhage (SAH) with Posterior Reversible Encephalopathy Syndrome]. All three of these cases were categorized as improved with EACA therapy. There were two cases that remained unchanged on EACA therapy (relapsed ALL patient with SDH and a relapsed AML patient with SAH). In both cases, the repeat neurologic imaging showed the ICH remained stable with EACA therapy. One patient worsened on EACA therapy. He was a 76-year-old male who received induction chemotherapy for myelodysplastic syndrome transformed to acute myeloid leukemia. He started EACA therapy after developing a SAH and repeat imaging demonstrated no change in the size of the SAH. However, three days after starting EACA therapy, he was incidentally found to have a sub segmental pulmonary embolism, EACA therapy was continued and he later succumbed to respiratory failure due to diffuse alveolar hemorrhage. There were four cases of Grade 3 bleeding categorized as "other." These were a spontaneous retroperitoneal hematoma, a psoas muscle hematoma, a fall injury causing facial and nasal lacerations and a fall causing a right upper extremity laceration and hematoma. All of this bleeding improved except the right upper extremity hematoma, which needed surgical debridement to prevent a compartment syndrome. Figure $2 \mathrm{~A}-2 \mathrm{C}$ depicts patients' bleeding grades, anatomic sites of bleeding and responses to EACA therapy. These data clearly demonstrate that, regardless of bleeding grade or bleeding site, the majority of patients responded to EACA therapy.

Table 3 Bleeding Sites (99 Patients)

\begin{tabular}{ll}
\hline Bleeding site & Patient (N) \\
\hline Spontaneous Skin Bleeding & 12 \\
Gastrointestinal & 23 \\
Pulmonary & 21 \\
Genito-Urinary & 13 \\
Ear, Nose, or Throat & 13 \\
Intracranial & 6 \\
Invasive Site bleeding* & 7 \\
Other** & 4 \\
\hline
\end{tabular}

* 5 patients had line insertion site bleeding ( 3 needed suturing to control the bleeding), I patient had breast biopsy site bleeding that needed suturing, and I patient had a burr hole site bleeding.

** One patient had each of the following: a spontaneous retroperitoneal hematoma; a psoas muscle hematoma; a facial and nasal laceration after a fall and a right upper extremity laceration and hematoma after a fall.

$2 \mathrm{~A}$

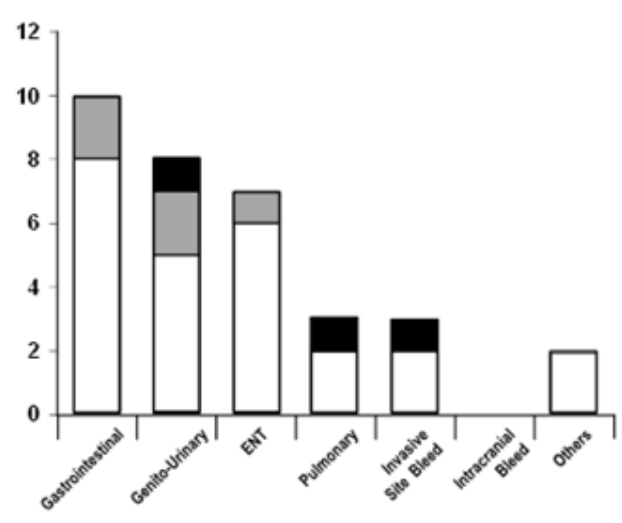

Figure 2A WHO grade2.

2B

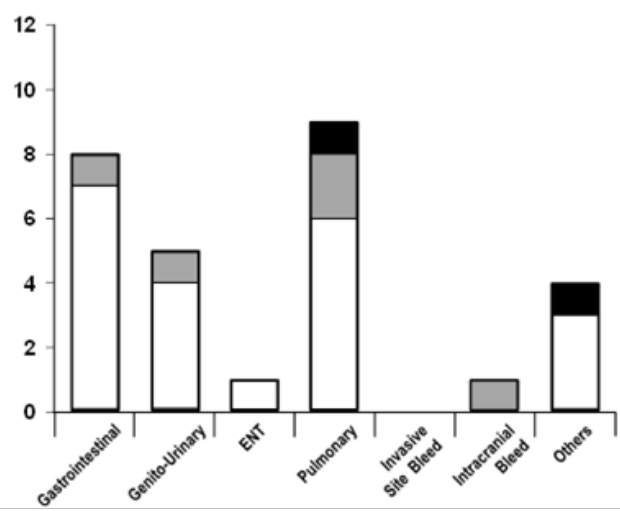

Figure 2B WHO grade3. 
2C

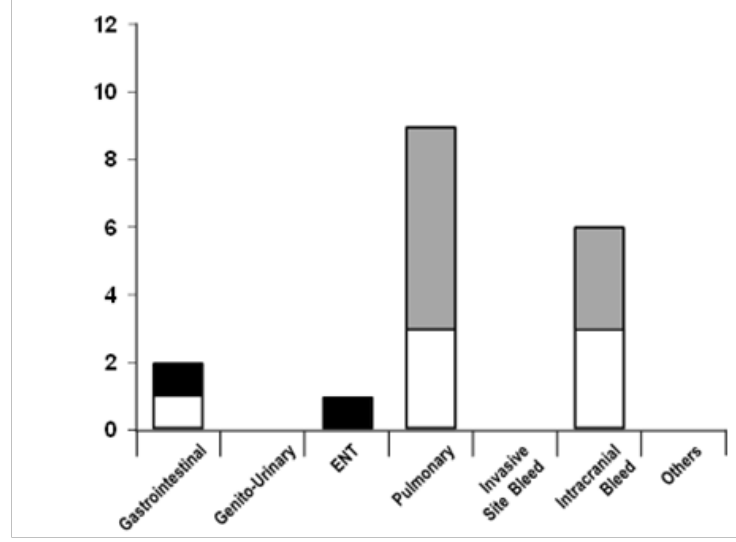

Figure 2CWho grade4.

Platelet refractoriness: Among the 99 patients, 48 patients were not platelet refractory. Of these patients, $69 \%$ showed improvement in their bleeding, $27 \%$ were unchanged and $4 \%$ had increased bleeding on EACA therapy. Among the 51 patients who were platelet refractory, $63 \%$ showed improvement in their bleeding, 20\% were unchanged and $12 \%$ increased their bleeding while on EACA therapy. Of the 51 refractory patients, $34(67 \%)$ were documented to have alloantibodies ( $>20 \%$ PRA positive), $10(20 \%$ ) had a PRA of $<20 \%$ PRA and $7(13 \%)$ patients were not tested for HLA alloantibodies. These data suggest that EACA therapy was equally effective regardless of whether the patient was or was not platelet refractory and regardless of the cause of their refractoriness; i.e., alloantibodies or other factors.

\section{Evaluation of non-bleeding patients}

Response to EACA therapy in patients treated prophylactically: Thirty-four patients $(26 \%)$ received EACA to prevent bleeding usually due to inadequate responses to their platelet transfusions. Their average age was 54 years, $47 \%$ were males and 11 patients were post HSCT $(32 \%)$. The median platelet count at the start of EACA therapy was $7,000 / \mu \mathrm{l}$ (range $4,700 / \mu \mathrm{l}-11,000 / \mu \mathrm{l}$ ) and median Hct was $26 \%$ (range $24 \%-28 \%$ ) and 11 patients were post HSCT (32\%). Daily doses of EACA in these patients ranged from 3 to 12grams (median 6grams) in 4 or 6 divided doses. By the end of the observation period, 31patients $(91 \%)$ treated prophylactically had not developed any evidence of bleeding. Three patients $(9 \%)$ developed Grade 1 bleeding and after increasing their dose of EACA, the bleeding stopped. One patient developed a clot in his bladder requiring bladder irrigation at which time EACA therapy was discontinued. This patient was found in retrospect to have had microscopic hematuria before starting EACA.

Platelet refractoriness: Among the 34patients, 23(68\%) were refractory to platelet transfusions. Of the 23 patients, 18 patients had PRA $>20 \%$, one was not tested and four had PRA $<20 \%$.

\section{Red cell and platelet transfusion requirements}

Per day of observation, there was an overall decrease in the use of both platelet and red blood cell transfusions after treatment with EACA compared to the number of pre-treatment transfusions. Changes in platelet usage are given as means and red cells as medians as the former were normally distributed and the latter were not. Among all 133 treated patients, the reduction in platelet usage was 0.25 units/ patient/day $(\mathrm{p}<0.003)$, while the decrease in red cell transfusions was 0.24 units/patient/day $(p<0.001)$ (Figure $3 A$ ). Among the $59 \%$ of patients treated for clinically significant bleeding (WHO Grade 2 or higher, $\mathrm{n}=78$ ), there was a reduction in platelet transfusions by 0.26 units/patient/day $(\mathrm{p}<0.01)$ and in red cell transfusions by 0.4 units/ patient/day $(p<0.001)$ (Figure 3B). A similar reduction in both types of transfusions was seen in the $34 \%$ of patients with severe bleeding (WHO Grade 3 or 4 bleeding, $n=45$ ) after treatment with EACA; i.e., platelet transfusions decreased by 0.3 units/patient/day, $(p=0.04)$ and red cell transfusions by 0.6 units/patient/day $(p<0.001)$ (Figure 3C). In the $49 \%$ of patients whose bleeding improved, the decrease in platelet transfusions was 0.33 units/patient/day $(\mathrm{p}=0.0006)$ and in red cell transfusions was 0.24 units/patient/day $(p<0.001)$. Even in the $20 \%$ of patients whose bleeding grades remained unchanged, platelet transfusions decreased by 0.3 units/patient/day $(\mathrm{p}=0.04)$ and red cell transfusions by 0.4 units/patient/day $(\mathrm{p}<0.001)$. In the $6 \%$ of patients whose bleeding worsened, there was an increase in transfusions. Platelet transfusions increased by 0.4 units/patient/day and red cell transfusions increased by 0.7 units/patient/day, but these increases did not attain statistical significance. In the $25 \%$ of patients who were treated with EACA prophylactically, there were no statistically significant changes in the use of either platelet or red cell transfusions.

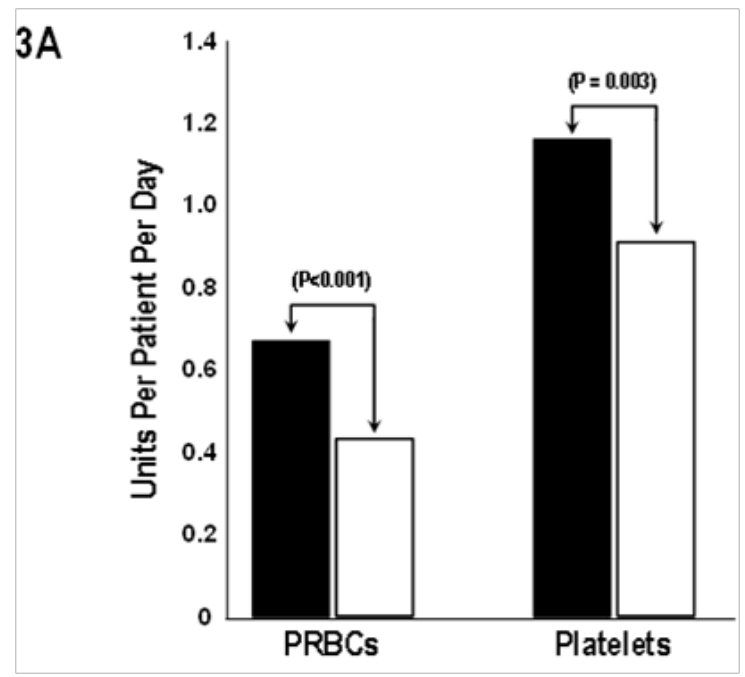

Figure 3A All Treated Patients $(n=\mid 33)$. PRBCs transfusions decreased by 0.24 units/patient/day and platelet transfusions by 0.25 units/patient/day after EACA therapy $(p<0.00 \mathrm{I}$ and $p=0.003$, respectively).

\section{Thrombotic complications}

Eight patients $(6 \%)$ developed a thrombosis. Incidental sub segmental pulmonary emboli were detected in three patients by a CT scan performed for other indications, two for evaluation of neutropenic fever and one for follow-up of a consolidation seen on a prior chest$\mathrm{X}$-ray. These three patients were not treated with anticoagulants. Two patients developed line-associated thrombus and were treated with anticoagulation. One patient developed a hemothorax (WHO Grade 3 bleed) due to a fall while on chronic warfarin therapy for a previous episode of bilateral deep venous thrombosis (DVT). Despite anticoagulation reversal, he showed radiographic evidence of an expanding hemothorax and required red blood cell transfusions. In this setting with persistent thrombocytopenia, the patient was treated with EACA for one week with cessation of bleeding. Two weeks later, he was diagnosed with a lower extremity DVT and 
warfarin anticoagulation was resumed. Another patient developed a femoral artery thrombus while on EACA therapy that required urgent thrombectomy and anticoagulation. One patient on prophylactic EACA therapy developed a clot in his bladder that required total bladder irrigation and discontinuation of EACA. No patient developed sinusoidal obstructive syndrome (veno-occlusive disease). ${ }^{15}$

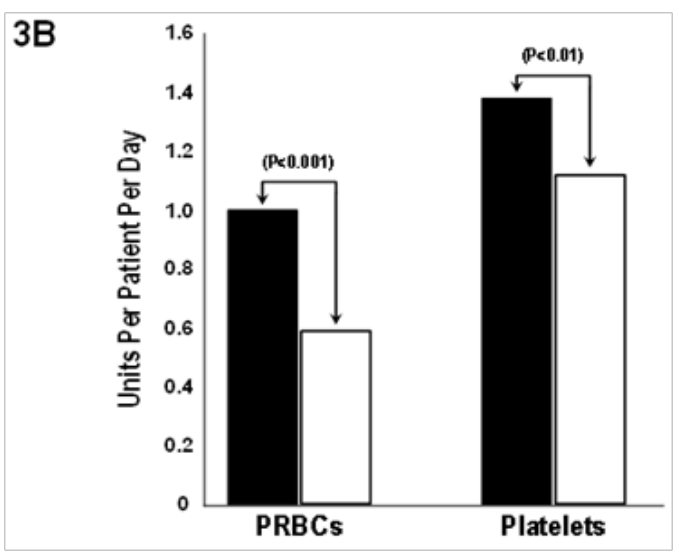

Figure 3B Patients with Grade2 or Higher Bleeding $(n=78)$. PRBCs transfusions decreased by 0.4 units/patient/day and platelet transfusions by 0.26 units/patient/day after EACA therapy $(p<0.001$ and $P<0.01$, respectively).

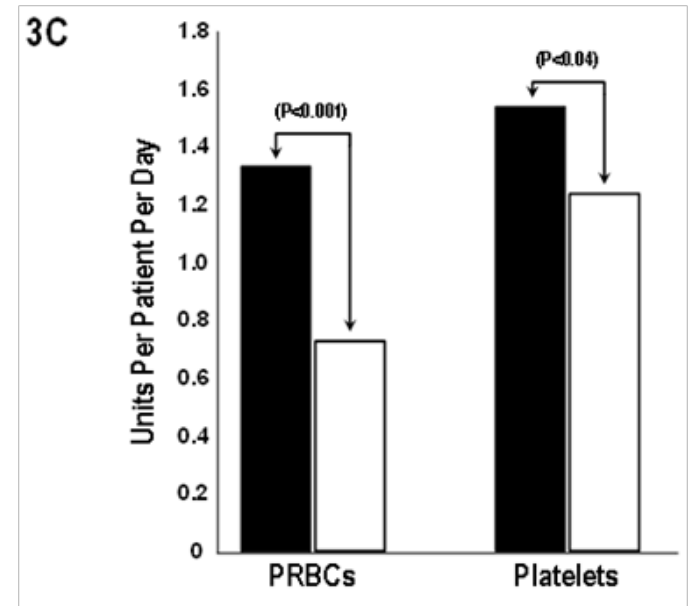

Figure 3C Patients with grade 3 or 4 bleeding $(n=45)$. PRBCs transfusions decreased by 0.6 units/patient/day and platelet transfusions by 0.3 units/patient/ day after EACA therapy $(p<0.001$ and $P<0.04$, respectively. Closed bars represent red cell and platelet transfusions pre-EACA therapy $(\boldsymbol{\square})$ and open bars post-EACA therapy $(\square)$.

\section{Conclusion}

A clot formed with inadequate platelets is friable, lyses rapidly and results in formation of a poor hemostatic plug. By preventing fibrinolysis, we postulate that EACA helps stabilize the clot and may have a role in preventing or controlling thrombocytopenic bleeding. To our knowledge, this report represents the largest retrospective analysis of EACA therapy given to patients with hypoproliferative thrombocytopenia. Ninety-nine patients were treated for active bleeding with EACA and the majority (65\%) demonstrated significant decreases in their bleeding following EACA therapy. Kalmadi et al. ${ }^{14}$ reported their experience with EACA therapy in 77 patients and showed a similar response rate with bleeding improvement in $66 \%$.
However, unlike the Kalmadi et al. ${ }^{14}$ study that found the patients with alloimmunization had a lower probability of response to EACA, we found that neither platelet alloimmunization nor any other factor (age, sex, diagnosis, baseline platelet counts and hematocrit or EACA dose) prevented patients from responding to EACA therapy. Importantly, in the 78 patients with clinically-significant WHO Grade 2 or greater bleeding, $68 \%$ improved and with WHO Grade 3 or greater bleeding, $62 \%$ improved $(75 \%$ with Grade 3 bleeding and $41 \%$ with Grade 4 bleeding). Of 6patients with intracranial bleeding, 3 improved, 3 stabilized and there were no deaths. Together with other smaller series, ${ }^{11-14}$ our data support the efficacy of EACA in treating thrombocytopenic bleeding. The decrease in red cell transfusions with EACA therapy likely resulted from less bleeding, whereas the decrease in platelet transfusions may indicate both less bleeding as well as a reduction in platelet consumption due to stabilization of clot formation. The smaller decrease in platelet transfusion requirements may also be related to the high rates of platelet refractoriness in our patient population resulting in little or no increase in platelet count with a transfusion and repeated transfusions for a specified platelet transfusion trigger, even with reduced bleeding. By decreasing both red blood cell and platelet transfusion needs, EACA therapy should significantly reduce transfusion costs.

EACA therapy also appears to be effective in preventing bleeding when used prophylactically. Of 34 patients treated prophylactically with EACA, mainly because of platelet refractoriness $(68 \%$ of patients), 91\% had no evidence of bleeding while 9\% developed Grade 1 bleeding that stopped following an increase in EACA dose. The use of EACA to prevent bleeding could make the practice of therapeutic-only platelet transfusions for patients with hematologic malignancies safer and more acceptable to treating clinicians. ${ }^{2,3} \mathrm{~A}$ decrease in bleeding risk and decreased transfusion requirements may also allow more patients to be managed as outpatients, significantly impacting the cost of care. A recent study reported the successful use of EACA therapy in 44 outpatients. ${ }^{16}$ In this report, the daily dose of EACA was 2 grams which was much lower than was used in our patients. Furthermore, only therapeutic platelet transfusions were given in this study that was performed exclusively in an outpatient setting and likely evaluated more stable patients. The authors reported 10 bleeding episodes in $16 \%$ of their patients. Two of those bleeds were higher than Grade 2 with one trauma-related intra cerebral bleed (Grade 4) and one bleed from a preexisting rectal ulcer (Grade 3). Prophylactic use of EACA in thrombocytopenia may represent a new treatment modality that might allow therapeutic-only platelet transfusions. Many physicians are reluctant to use anti-fibrinolytic therapy due to a perceived risk of thromboembolism. The incidence of thromboembolism in hospitalized neutropenic patients with acute leukemia has previously been reported as $6.3 \%$ based on ICD- 9 codes from the discharge database.${ }^{17}$ In contrast, we reviewed medical charts and radiology imaging reports for any evidence of thrombosis. In our cohort, we reported a rate of $6 \%$ of which two events may not have been reported if thromboembolic events were collected by the same criteria as used in the prior report. We could not attribute additional thromboembolic risk to EACA use in our study cohort. This study was limited by its retrospective and observational status. These results support the need for a prospective randomized placebo controlled trial to determine the true efficacy and safety of EACA therapy in patients with hypoproliferative thrombocytopenia due to chemotherapy/ radiation or hematopoietic stem cell transplantation for hematologic malignancies. In patients with hypoproliferative thrombocytopenia, 
EACA therapy appears to be effective in both treating and preventing bleeding events. EACA therapy results in very significant reductions in both red blood cell and platelet transfusions and appears to be well tolerated with no obvious increase in thrombotic events. Larger prospective randomized controlled clinical trials need to be conducted to evaluate both efficacy and safety of EACA therapy in hypoproliferative thrombocytopenic patients.

\section{Acknowledgements}

We would like to acknowledge Ginny Knight and Betsy Lusby for providing the administrative support of preparing and submitting this manuscript.

\section{Conflict of interest}

The author declares no conflict of interest.

\section{References}

1. Miller AB, Hoogstraten B, Staquet M, et al. Reporting the results of cancer treatment. Cancer. 1981;47(1):207-214.

2. Stanworth SJ, Estcourt LJ, Powter G, et al. A no-prophylaxis platelet-transfusion strategy for hematologic cancers. $N$ Engl J Med. 2013;368(19):1771-1780.

3. Slichter SJ, Kaufman RM, Assmann SF, et al. Dose of prophylactic platelet transfusions and prevention hemorrhage. $N$ Engl $J$ Med. 2010;362(7):600-613.

4. Wandt H, Eckart KS, Wendelin K, et al. Therapeutic platelet transfusion versus routine prophylactic transfusion in patients with haematological malignancies: an open-label, multicentre, randomised study. Lancet. 2012;380(9850):1309-1316.

5. Rebulla P, Finazzi G, Marangoni F, et al. The threshold for prophylactic platelet transfusions in adults with acute myeloid leukemia. $N$ Engl $J$ Med. 1997;337(26):1870-1875.

6. Wandt H, Frank M, Ehninger G, et al. Safety and cost effectiveness of a 10x10(9)/L trigger for prophylactic platelet transfusions compared with traditional $20 \times 10(9) / \mathrm{L}$ trigger: a prospective comparative trial in 105 patients with acute myeloid leukemia. Blood. 1998;91(10):3601-3606.
7. Verstraete M. Clinical application of inhibitors of fibrinolysis. Drugs. 1985;29(3):236-261.

8. Henry DA, Carless PA, Moxey AJ, et al. Anti-fibrinolytic use for minimising perioperative allogeneic blood transfusion. Cochrane Database Syst Rev. 2011;1:CD001886.

9. Nichols WL, Hultin MB, James AH, et al. von Willebrand disease (vWD): evidence-based diagnosis and management guidelines, the National Heart, Lung and Blood Institute (NHLBI) Expert Panel Report (USA). Haemophilia. 2008;14(2):171-232.

10. Ghosh K, Shetty S, Jijina F, et al. Role of epsilon amino caproic acid in the management of haemophilic patients with inhibitors. Haemophilia. 2004;10(1):58-62.

11. Gallardo RL, Gardner FH. Aminocaproic acid for bleeding in thrombocytopenic patients. Tex Med. 1985;81(1):30-32.

12. Bartholomew JR, Salgia R, Bell WR. Control of bleeding in patients with immune and nonimmune thrombocytopenia with aminocaproic acid. Arch Intern Med. 1989;149(9):1959-1961.

13. Garewal HS, Durie BG. Anti-fibrinolytic therapy with aminocaproic acid for control of bleeding in thrombocytopenic patients. Scand J Haematol. 1985;35(5):497-500

14. Kalmadi S, Tiu R, Lowe C, et al. Epsilon aminocaproic acid reduces transfusion requirements in patients with thrombocytopenic hemorrhage. Cancer. 2006;107(1):136-140.

15. Imran H, Tleyjeh IM, Zirakzadeh A, et al. Use of prophylactic anticoagulation and the risk of hepatic veno-occlusive disease in patients undergoing hematopoietic stem cell transplantation: a systematic review and meta-analysis. Bone Marrow Transplant. 2006;37(7):677-686.

16. Antun AG, Gleason S, Arellano M, et al. Epsilon aminocaproic acid prevents bleeding in severely thrombocytopenic patients with hematological malignancies. Cancer. 2013;119(21):3784-3787.

17. Khorana AA, Francis CW, Culakova E, et al. Thromboembolism in hospitalized neutropenic cancer patients. J Clin Oncol. 2006;24(3):484490 . 